\title{
Análise da distribuição de médicos especialistas em microrregiões de saúde na área de consórcios intermunicipais de saúde no Sul de Minas Gerais
}

\author{
Analysis of the distribution of doctors specialized in health micro-regions in the area of \\ intermunicipal health consortia in the South of Minas Gerais
}
Análisis de la distribución de médicos especializados en microrregiones de salud en el área de consorcios intermunicipales de salud en el Sur de Minas Gerais

Eduardo Gonçalves ${ }^{1}$, Alcir José de Oliveira Júnior ${ }^{1}$, Fábio Luiz Mialhe ${ }^{1 *}$.

\begin{abstract}
RESUMO
Objetivo: Analisar a distribuição de profissionais médicos especialistas em cinco microrregiões assistenciais na área de abrangência de consórcios intermunicipais de saúde no Sul de Minas Gerais. Métodos: Trata-se de um estudo transversal descritivo, utilizando-se de dados secundários de acesso amplo, irrestrito e público, a partir de dados constantes na base de dados do Cadastro Nacional de Estabelecimentos de Saúde (CNES), na competência dezembro de 2018. Para a coleta dos dados, utilizou-se os arquivos disponíveis pelo sítio do Datasus, referente a base do CNES e, posteriormente, tabulados por meio do programa Tabnet e Tabwin. Resultados: Houve grande variabilidade entre as microrregiões na área de abrangência dos consórcios intermunicipais de saúde existentes quanto a distribuição de profissionais especialistas dentro de uma mesma macrorregião, com ênfase para algumas especialidades que indicam maior dificuldade de acesso por meio do Sistema Único de Saúde. Conclusão: Os resultados apresentados são fundamentais para subsidiarem as discussões e elaboração de políticas públicas que possam contribuir para a equidade assistencial em cada região de saúde, uma vez que relacionados à Consórcios Intermunicipais de Saúde, não foram encontrados estudos anteriores que apresentem esta abordagem, distanciando, portanto, da prática assistencial diária das gestões municipais.
\end{abstract}

Palavras-chave: Consórcios de saúde, Planejamento em saúde, Gestão em saúde, Regionalização.

\begin{abstract}
Objective: To analyze the distribution of specialist medical professionals in five care micro-regions in the coverage area of intermunicipal health consortia in the south of Minas Gerais. Methods: This is a descriptive cross-sectional study, using secondary data with broad, unrestricted and public access, based on data contained in the database of the National Registry of Health Facilities (NRHF), in the December 2018 competence. For data collection, we used the files available on the Datasus website, referring to the NRHF database and, later, tabulated through the program Tabnet and Tabwin. Results: There was great variability among the micro-regions in the coverage area of the existing inter-municipal health consortia regarding the distribution of specialist professionals within the same macro-region, with an emphasis on some specialties that indicate greater difficulty of access through the Unified Health System. Conclusion: The results presented are fundamental to support the discussions and elaboration of public policies that can contribute to the equity of assistance in each health region, since related to the Intermunicipal Health Consortia, no previous studies were found that present this approach, distancing, therefore, the daily care practice of municipal administrations.
\end{abstract}

Keywords: Health consortia, Health planning, Health management, Regional health planning.

${ }^{1}$ Faculdade de Odontologia de Piracicaba (FOP), Universidade Estadual de Campinas (Unicamp), Piracicaba - SP. *E-mail: mialhe@unicamp.br

SUBMETIDO EM: $3 / 2021$ 


\section{RESUMEN}

Objetivo: Analizar la distribución de profesionales médicos especialistas en cinco microrregiones de atención en el área de cobertura de los consorcios de salud intermunicipales en el sur de Minas Gerais. Métodos: Se trata de un estudio descriptivo de corte transversal, utilizando datos secundarios con acceso amplio, irrestricto y público, con base en datos contenidos en la base de datos del Registro Nacional de Establecimientos de Salud (RNES), en la competencia diciembre 2018. Para la recolección de datos, utilizamos los archivos disponibles en el sitio web de Datasus, haciendo referencia a la base de datos RNES y, posteriormente, tabulados a través del programa Tabnet y Tabwin. Resultados: Hubo gran variabilidad entre las microrregiones en el área de cobertura de los consorcios de salud intermunicipales existentes en cuanto a la distribución de profesionales especialistas dentro de la misma macrorregión, con énfasis en algunas especialidades que indican mayor dificultad de acceso a través del Sistema Unificado. Sistema de Salud. Conclusión: Los resultados presentados son fundamentales para sustentar las discusiones y elaboración de políticas públicas que puedan contribuir a la equidad asistencial en cada región de salud, ya que relacionados con los Consorcios Intermunicipales de Salud, no se encontraron estudios previos que presenten este abordaje, distanciamiento, por tanto, la práctica asistencial diaria de las administraciones municipales.

Palabras clave: Consorcios de salud, Planificación en salud, Gestión en salud, Regionalización.

\section{INTRODUÇÃO}

A escassez de médicos em atuação nos sistemas de saúde é apontada, na maioria dos países, como um problema grave e persistente ao longo dos últimos anos, cujo enfrentamento é constante por meio de variadas estratégias (ORGANIZAÇÃO MUNDIAL DA SAÚDE, 2012). No Brasil, este cenário também se reproduz e, é evidenciado por meio de alguns estudos que apontam a falta de médicos em partes do território nacional (GIRARDI SN, et al., 2011; UNIVERSIDADE FEDERAL DE MINAS GERAIS, 2014).

A fim de superar essas dificuldades, a busca pelo associativismo municipal predomina mediante a intencionalidade de respostas frente à responsabilização sanitária apresentada pelo processo de descentralização ao longo dos últimos anos, desta forma, quando o poder e as responsabilidades são atribuídas sobremaneira aos municípios, são expostos, da mesma forma, os desafios para a consolidação de parcerias que possam refletir na resolução de problemas comuns da sociedade, para que assim, permitam o enfrentamento de dificuldades relacionadas à escassez de recursos financeiros, materiais e o provimento de recursos humanos para a efetivação da assistência à saúde dentro dos princípios da universalidade, integralidade, equidade, uma vez que representam uma estratégia importante para a superação dos efeitos adversos do protagonismo por este ente federado (GRZELZAK M, et al., 2017, 2018; MENDES W, et al., 2019; JULIÃO KS e OLIVEIRA C, 2020).

Para mais, constata-se que, um dos maiores desafios da gestão municipal, quando se refere a necessidade da oferta de serviços de média e alta complexidade ambulatoriais tais como procedimentos, consultas, exames e cirurgias, é na busca de respostas às demandas e sua consonância com prestadores disponíveis para a assistência em cada localidade. Do mesmo modo, a confederação nacional de municípios apresenta os consórcios intermunicipais de saúde como potenciais dispositivos para as soluções coletivas de problemas individualizados, otimizando custos e aumentando o escopo das possibilidades para cada membro consorciado, uma vez que são iniciativas autônomas de municípios localizados em uma mesma área geográfica, dispostos à pactuação intergestores, visando a gestão de serviços especializados e apoio diagnóstico, cujos impactos interessam diretamente aos seus munícipes (CONFEDERAÇÃO NACIONAL DE MUNICÍPIOS, 2016).

Outrossim, no Brasil, a oferta de serviços de média complexidade constitui-se em um grande vazio assistencial, sendo um dos maiores pontos de estrangulamento do sistema de saúde (LIEBEL G, 2016). Diante disso, é fundamental o entendimento de fatores que possam impactar diretamente a aplicabilidade de recursos por meio de consórcios de saúde, empreendendo esforços regionais para melhor alocação de recursos financeiros que remontem respostas adequadas às necessidades apresentadas. Com efeito, a viabilidade de arranjos, como a aplicação do consórcio público, ganha conotação especial, pois possibilita segurança jurídica, fidedignidade sobre os contratos estabelecidos, e menor tendência às mudanças 
ocasionadas pelas alternâncias políticas-partidárias, apesar disso, um estudo indicou que os consórcios se tornam instrumentos comuns dos atores envolvidos, mas, dificilmente dos interesses coletivos da totalidade territorial abrangida (LIEBEL G, 2016).

Dado o fato de que, o processo de descentralização emanado pela constituinte não apresentou mecanismos efetivos de coordenação, os quais permitissem a gerência de ações e serviços com qualidade, sendo assim, a agenda relacionada a cooperação federativa vem ganhando significância nos últimos anos no campo político e das pesquisas (LEAL EMM, 2019). Porém, considerando o atual cenário de crise, já identificado nas relações federalistas, como o subfinanciamento federal crônico e histórico do Sistema Único de Saúde, acrescido da consolidação da responsabilização municipal das ações e serviços de saúde, é possível perceber o aumento exponencial dos gastos em saúde pela municipalidade (ARAÚJO CEL, et al., 2017; BRASIL, 2017; CONTARATO PC, et al., 2019; FUNCIA FR, 2019).

Neste ínterim processo de regionalização ou compartilhamento coletivo, deve-se compreender a organização das ações e serviços de saúde em uma região, objetivando a integralidade do cuidado, otimização na alocação dos recursos e a redução das iniquidades (MEDEIROS CRG, et al., 2017). Deste modo, os consórcios foram então reconhecidos como potenciais dispositivos estratégicos, como uma alternativa para a organização regional das demandas, uma vez que a regionalização passou a ser compreendida como uma fase incremental das atribuições da descentralização (CARVALHO ALB, et al., 2017).

Santos L e Campos GWS (2015) afirmaram que o único caminho para o Sistema Único de Saúde ser inteiramente nacional é regionalizá-lo, fomentando as regiões de saúde com instrumentos para a gestão compartilhada, interfederativa e responsável. Nesta perspectiva, estudos indicaram a necessidade de adoção de políticas públicas que possam impactar na redução das iniquidades entre os municípios do país, das quais remetam à possibilidade de oferta de tecnologias de qualificação da gestão municipal, otimizando a aplicabilidade dos recursos disponíveis, a fim de integrar e coordenar ações e serviços em distintos espaços geográficos, com as necessidades em escalas de qualidade e custos pertinentes (SANTOS-NETO JA, et al., 2019; FLEXA RGC e BARBASTEFANO RG, 2020).

Logo, busca-se compreender com maior precisão esse cenário, necessitando-se de estudos que possibilitem análises profundas das regiões de saúde quanto à existência e a distribuição geográfica de profissionais, como médicos especialistas, cujas vinculações podem remeter a potenciais respostas para a superação de vazios assistenciais, com ênfase nas relações consorciadas. A exploração da temática se apresenta como estratégia primordial para as políticas públicas de saúde, visto que, estes fenômenos poderão ser capazes de traduzir as disparidades de valores financeiros adotados em cada um dos consórcios em uma mesma macrorregião de saúde. Sendo assim, este estudo objetivou analisar a distribuição de profissionais especialistas em áreas de abrangência de Consórcios Intermunicipais de Saúde no Sul do Estado de Minas Gerais no ano de 2018.

\section{MÉTODOS}

Trata-se de um estudo transversal descritivo, utilizando-se de dados secundários de acesso amplo, irrestrito e público, a partir de dados constantes na base de dados do Cadastro Nacional de Estabelecimentos de Saúde (CNES), na competência dezembro de 2018. Para a coleta dos dados foi utilizado os arquivos disponíveis para tabulação originados pelo CNES no sítio do Datasus, sistema este que abarca em sua base dados cadastrais de estabelecimentos e profissionais de saúde públicos e privados, tabulados por meio do programa Tabnet e Tabwin (BRASIL, 2020).

Para tal, buscou-se identificar o código brasileiro de ocupação de cada especialidade médica constante na Portaria № 1.631, de 1ํ de outubro de 2015 que aprovou os critérios e parâmetros para o planejamento e programação de ações e serviços de saúde no âmbito do Sistema Único de Saúde (SUS), a saber: angiologia, cirurgia vascular, cardiologia, dermatologia, endocrinologia, gastroenterologia, proctologia, neurologia, neurocirurgia, neurofisiologia, oftalmologia, ortopedia, otorrinolaringologia, pneumologia, urologia e reumatologia. Com base nestes dados, calculou-se a proporcionalidade de médicos por habitantes em cada 
uma das especialidades elencadas disponíveis para o SUS e na rede privada em cada uma das microrregiões de abrangência dos consórcios identificados nesta mesma macrorregião de saúde (BRASIL, 2015). Foram analisados os dados referentes a 5 microrregiões de saúde da área de abrangência de 4 Consórcios Intermunicipais de saúde no Sul do Estado de Minas Gerais. A população estimada para o conjunto destas microrregiões do presente estudo se apresenta em 855.140 habitantes conforme estimativa do TCU em 2008.

Após a captação dos dados dos bancos de dados do CNES os mesmos foram exportados para o programa Microsoft Office Excel, versão 2010 (Microsoft Corp.), para a consolidação, ajustes e congregação das variáveis em análise, agrupando-se as especialidades por ordem alfabética, aplicando-se a razão de médicos para cada 100 mil habitantes, considerando a população de cada microrregião de saúde em estudo. Logo, a razão de profissionais médicos quanto à necessidade de cada microrregião, considerou-se os parâmetros por especialidade, apresentados por meio da Portaria № 1.631, de 1ำ de outubro de 2015. Nas colunas subsequentes, foi possível apresentar a existência de profissionais médicos por especialidade, público e privado, demonstrando-se ao final o resultado da soma destes, menos o percentual referente à necessidade para cada território microrregional.

\section{RESULTADOS}

A Tabela 1 apresenta a distribuição de profissionais especialistas na microrregião de Lavras-MG, cuja população perfaz cerca de 169.514 habitantes conforme estimativa do Tribunal de Contas da União em 2008, bem como a razão médico/100 mil habitantes, razão médico/necessidade da microrregião e a frequência dos profissionais dispostos no banco de dados do CNES com vínculo SUS e privado. Esta microrregião não apresentou a existência de profissionais especialistas atuando com vínculo para o SUS para $56,25 \%$ das especialidades elencadas.

Tabela 1 - Distribuição de profissionais de diferentes especialidades médicas na microrregião de Lavras com vínculo SUS e privado versus a avaliação da necessidade em relação ao parâmetro constante no Caderno de critérios e parâmetros SUS.

\begin{tabular}{lccccc}
\multicolumn{1}{c}{$\begin{array}{c}\text { Microrregião de } \\
\text { Lavras }\end{array}$} & $\begin{array}{c}\text { Razão médico/ } \\
\mathbf{1 0 0} \text { mil habitantes } \\
\text { (A) }\end{array}$ & $\begin{array}{c}\text { Razão médico/ } \\
\text { Necessidade da } \\
\text { microrregião (B) }\end{array}$ & $\begin{array}{c}\text { Existente } \\
\text { SUS (C) }\end{array}$ & $\begin{array}{c}\text { Existente } \\
\text { privado (D) }\end{array}$ & $\begin{array}{c}\text { Resultado } \\
\text { (C+D-B) }\end{array}$ \\
\hline Angiologia & 1,5 & 2,54 & 4 & 4 & 5,46 \\
Cirurgia Vascular & 1,5 & 2,54 & 0 & 0 & $-2,54$ \\
Cardiologia & 6,5 & 11,02 & 15 & 4 & 7,98 \\
Dermatologia & 2,3 & 3,9 & 5 & 4 & 5,1 \\
Endocrinologia & 1,5 & 2,54 & 0 & 2 & $-0,54$ \\
Gastroenterologia & 2,5 & 4,24 & 0 & 0 & $-4,24$ \\
Proctologia & 2 & 3,39 & 0 & 0 & $-3,39$ \\
Neurologia & 2,6 & 5,93 & 7 & 2 & 3,07 \\
Neurocirurgia & 3,5 & 5,93 & 0 & 0 & $-5,93$ \\
Neurofisiologia & 3,5 & 5,93 & 0 & 0 & $-5,93$ \\
Oftalmologia & 4 & 6,78 & 5 & 1 & $-0,78$ \\
Ortopedia & 10 & 16,95 & 1 & 2 & $-13,95$ \\
Otorrino & 3 & 5,09 & 3 & 0 & $-2,09$ \\
Pneumologia & 3 & 5,09 & 0 & 0 & $-5,09$ \\
Urologia & 3 & 5,09 & 0 & 0 & $-5,09$ \\
Reumatologia & 1 & 1,7 & 0 & 0 & $-1,7$ \\
\hline
\end{tabular}

Fonte: Gonçalves E, et al., 2021; dados extraídos do Datasus (BRASIL, 2020). 
Quando se avalia a especialidade com a maior concentração de profissionais médicos, evidencia-se a cardiologia $(7,98 \%)$ com expressiva composição de médicos tanto para o SUS quanto para o setor privado. As especialidades: dermatologia $(5,1 \%)$ e neurologia $(3,07 \%)$ apresentam índice positivo para a necessidade da microrregião em relação ao parâmetro disposto na Portaria № 1635 de $1^{\circ}$ de outubro de 2015, quando considerados os valores apresentados para ambas as vinculações. Por fim, a oftalmologia $(-0,78 \%)$, ortopedia $(-13,95 \%)$ e otorrinolaringologia $(-2,09 \%)$ apresentam índices negativos mesmo quando da agregação dos vínculos SUS e privado.

A Tabela 2 apresenta a distribuição de profissionais especialistas na microrregião de Três Corações-MG, cuja população perfaz cerca de 127.094 habitantes conforme estimativa do Tribunal de Contas da União em 2008. Esta microrregião não apresentou em nenhum vínculo possível, profissionais especialistas: cirurgião vascular, proctologia, neurocirurgião, neurofisiologia e pneumologista, totalizando $31,25 \%$ do total das especialidades do estudo. Índices apresentados abaixo do parâmetro constante como indicativo da necessidade de médicos para a microrregião nas seguintes especialidades: dermatologia $(-0,92 \%)$, endocrinologia $(-0,91 \%)$, gastroenterologia $(-2,18 \%)$, oftalmologia $(-0,08 \%)$, ortopedia $(-8,71 \%)$, otorrinolaringologia $(-2,81 \%)$, urologia $(-2,81 \%)$ e reumatologia $(-0,27 \%)$. Na análise a maior diferença apurada entre as 16 especialidades para a microrregião sem distinção de vinculação se deu na angiologia $(2,09)$.

Tabela 2 - Distribuição de profissionais de diferentes especialidades médicas na microrregião de Três Corações com vínculo SUS e privado versus a avaliação da necessidade em relação ao parâmetro constante no Caderno de critérios e parâmetros SUS.

\begin{tabular}{lccccc}
\hline $\begin{array}{c}\text { Microrregião de Três } \\
\text { Corações }\end{array}$ & $\begin{array}{c}\text { Razão médico/ } \\
\mathbf{1 0 0} \text { mil } \\
\text { habitantes (A) }\end{array}$ & $\begin{array}{c}\text { Razão médico/ } \\
\text { Necessidade da } \\
\text { microrregião (B) }\end{array}$ & $\begin{array}{c}\text { Existente } \\
\text { SUS (C) }\end{array}$ & $\begin{array}{c}\text { Existente } \\
\text { privado (D) }\end{array}$ & $\begin{array}{c}\text { Resultado } \\
\text { (C+D-B) }\end{array}$ \\
\hline Angiologia & 1,5 & 1,91 & 4 & 0 & 2,09 \\
Cirurgia Vascular & 1,5 & 1,91 & 0 & 0 & $-1,91$ \\
Cardiologia & 6,5 & 8,26 & 7 & 3 & 1,74 \\
Dermatologia & 2,3 & 2,92 & 2 & 0 & $-0,92$ \\
Endocrinologia & 1,5 & 1,91 & 1 & 0 & $-0,91$ \\
Gastroenterologia & 2,5 & 3,18 & 0 & 1 & $-2,18$ \\
Proctologia & 2 & 2,54 & 0 & 0 & $-2,54$ \\
Neurologia & 3,5 & 4,45 & 5 & 1 & 1,55 \\
Neurocirurgia & 3,5 & 4,45 & 0 & 0 & $-4,45$ \\
Neurofisiologia & 3,5 & 4,45 & 0 & 0 & $-4,45$ \\
Oftalmologia & 4 & 5,08 & 3 & 2 & $-0,08$ \\
Ortopedia & 10 & 12,71 & 4 & 0 & $-8,71$ \\
Otorrino & 3 & 3,81 & 1 & 0 & $-2,81$ \\
Pneumologia & 3 & 3,81 & 0 & 0 & $-3,81$ \\
Urologia & 3 & 3,81 & 1 & 0 & $-2,81$ \\
Reumatologia & 1 & 1,27 & 1 & 0 & $-0,27$ \\
\hline
\end{tabular}

Fonte: Gonçalves E, et al., 2021; dados extraídos do Datasus (BRASIL, 2020).

A Tabela 3 apresenta a distribuição de profissionais especialistas na microrregião de Varginha-MG, cuja população perfaz cerca de 180.733 habitantes, sendo o município de Varginha-MG (136.602 hab.) o maior 
entre todos os municípios compreendidos nas microrregiões conforme estimativa do IBGE 2020. A microrregião não apresentou alocação de profissionais especialistas para 31,25\% das especialidades. Ênfase para a especialidade cardiologia com índice positivo apresentado (19,25\%) acima do parâmetro sugerido para a necessidade do referido território assim como para o índice negativo apresentado pela ortopedia (-15,07\%) abaixo da necessidade parametrizada. Destaca-se também o provimento maior de profissionais dermatologistas com vinculações para o setor privado (91\%) em detrimento do SUS (9\%). Índices abaixo do parâmetro/razão médico necessidade da microrregião foram identificados para a gastroenterologia $(-0,52)$, neurologia $(-1,33)$, otorrinolaringologia $(-1,42)$, pneumologia $(-3,42)$, urologia $(-4,42)$, com acentuada diferença para a ortopedia $(-15,07)$.

Tabela 3 - Distribuição de profissionais de diferentes especialidades médicas na microrregião de Varginha com vínculo SUS e privado versus a avaliação da necessidade em relação ao parâmetro constante no Caderno de critérios e parâmetros SUS.

\begin{tabular}{lccccc}
\hline $\begin{array}{l}\text { Microrregião de } \\
\text { Varginha }\end{array}$ & $\begin{array}{c}\text { Razão médico/ } \\
\text { 100 mil } \\
\text { habitantes (A) }\end{array}$ & $\begin{array}{c}\text { Razão médico/ } \\
\text { Necessidade da } \\
\text { microrregião (B) }\end{array}$ & $\begin{array}{c}\text { Existente } \\
\text { SUS (C) }\end{array}$ & $\begin{array}{c}\text { Existente } \\
\text { privado (D) }\end{array}$ & $\begin{array}{c}\text { Resultado } \\
\text { (C+D-B) }\end{array}$ \\
\hline Angiologia & 1,5 & 2,71 & 5 & 1 & 3,29 \\
Cirurgia Vascular & 1,5 & 2,71 & 0 & 0 & $-2,71$ \\
Cardiologia & 6,5 & 11,75 & 27 & 4 & 19,25 \\
Dermatologia & 2,3 & 4,16 & 1 & 10 & 6,84 \\
Endocrinologia & 1,5 & 2,71 & 0 & 5 & 2,29 \\
Gastroenterologia & 2,5 & 4,52 & 2 & 2 & $-0,52$ \\
Proctologia & 2 & 3,61 & 0 & 0 & $-3,61$ \\
Neurologia & 3,5 & 6,33 & 5 & 0 & $-1,33$ \\
Neurocirurgia & 3,5 & 6,33 & 0 & 0 & $-6,33$ \\
Neurofisiologia & 3,5 & 6,33 & 0 & 0 & $-6,33$ \\
Oftalmologia & 4 & 7,23 & 3 & 5 & 0,77 \\
Ortopedia & 10 & 18,07 & 2 & 1 & $-15,07$ \\
Otorrino & 3 & 5,42 & 3 & 1 & $-1,42$ \\
Pneumologia & 3 & 5,42 & 0 & 2 & $-3,42$ \\
Urologia & 3 & 5,42 & 1 & 0 & $-4,42$ \\
Reumatologia & 1 & 1,81 & 0 & 0 & $-1,81$ \\
\hline
\end{tabular}

Fonte: Gonçalves E, et al., 2021; dados extraídos do Datasus (BRASIL, 2020).

A Tabela 4 apresenta a distribuição de profissionais especialistas na microrregião de São Lourenço-MG, cuja população é de 256.100 habitantes segundo estima do TCU 2008, sendo também a maior microrregião da macrorregião representada pelo estudo, composta por 24 municípios. Destaca-se nesta microrregião a especialidade oftalmologia com índice de 13,76\% superior ao parâmetro recomendado para o território microrregional, a cardiologia com $11,35 \%$ e a neurologia com $8,35 \%$. Apresentaram índices negativos na soma da disponibilidade para o SUS e setor privado: endocrinologia $(-1,84 \%)$, neurocirurgia $(-4,65 \%)$, ortopedia $(-11,61 \%)$, otorrinolaringologia $(-2,68 \%)$, urologia $(-5,68 \%)$ e reumatologia $(-1,56 \%)$. Não foram identificadas as seguintes especialidades existentes nos bancos de dados da microrregião: cirurgião vascular, gastroenterologia, proctologia, neurofisiologia, pneumologia, representando $31,25 \%$ das demais especialidades. 
Tabela 4 - Distribuição de profissionais de diferentes especialidades médicas na microrregião de São Lourenço com vínculo SUS e privado versus a avaliação da necessidade em relação ao parâmetro constante no Caderno de critérios e parâmetros SUS.

\begin{tabular}{lccccc}
\hline $\begin{array}{c}\text { Microrregião de São } \\
\text { Lourenço }\end{array}$ & $\begin{array}{c}\text { Razão médico/ } \\
\mathbf{1 0 0} \text { mil } \\
\text { habitantes (A) }\end{array}$ & $\begin{array}{c}\text { Razão médico/ } \\
\text { Necessidade da } \\
\text { microrregião (B) }\end{array}$ & $\begin{array}{c}\text { Existente } \\
\text { SUS (C) }\end{array}$ & $\begin{array}{c}\text { Existente } \\
\text { privado (D) }\end{array}$ & $\begin{array}{c}\text { Resultado } \\
\text { (C+D-B) }\end{array}$ \\
\hline Angiologia & 1,5 & 3,84 & 3 & 1 & 0,16 \\
Cirurgia Vascular & 1,5 & 3,84 & 0 & 0 & $-3,84$ \\
Cardiologia & 6,5 & 16,65 & 21 & 7 & 11,35 \\
Dermatologia & 2,3 & 5,89 & 2 & 7 & 3,11 \\
Endocrinologia & 1,5 & 3,84 & 2 & 0 & $-1,84$ \\
Gastroenterologia & 2,5 & 6,4 & 0 & 0 & $-6,4$ \\
Proctologia & 2 & 5,12 & 0 & 0 & $-5,12$ \\
Neurologia & 3,5 & 6,65 & 13 & 2 & 8,35 \\
Neurocirurgia & 3,5 & 6,65 & 2 & 0 & $-4,65$ \\
Neurofisiologia & 3,5 & 6,65 & 0 & 0 & $-6,65$ \\
Oftalmologia & 4 & 10,24 & 23 & 1 & 13,76 \\
Ortopedia & 10 & 25,61 & 14 & 0 & $-11,61$ \\
Otorrino & 3 & 7,68 & 4 & 1 & $-2,68$ \\
Pneumologia & 3 & 7,68 & 0 & 0 & $-7,68$ \\
Urologia & 3 & 7,68 & 1 & 1 & $-5,68$ \\
Reumatologia & 1 & 2,56 & 0 & 1 & $-1,56$ \\
\hline
\end{tabular}

Fonte: Gonçalves E, et al., 2021; dados extraídos do Datasus (BRASIL, 2020).

A Tabela 5 apresenta a distribuição de profissionais especialistas na microrregião de Três Pontas-MG, com uma população 169.514 habitantes. Esta microrregião apresentou dados negativos quanto à existência de profissionais especialistas em 12 das 16 especialidades contempladas no estudo (75\%). Em 8 destas especialidades não foi possível identificar profissionais disponíveis em nenhum dos vínculos abordados: SUS e privado. Apenas as especialidades: angiologia $(5,46 \%)$, cardiologia $(7,98 \%)$, dermatologia $(5,1 \%)$, neurologia (3,07\%) apresentaram índices acima do parâmetro identificado como necessidade para a microrregião.

Tabela 5 - Distribuição de profissionais de diferentes especialidades médicas na microrregião de Lavras com vínculo SUS e privado versus a avaliação da necessidade em relação ao parâmetro constante no Caderno de critérios e parâmetros SUS.

\begin{tabular}{lccccc}
\hline $\begin{array}{c}\text { Microrregião de } \\
\text { Três Pontas }\end{array}$ & $\begin{array}{c}\text { Razão } \\
\text { médico/ } \\
\mathbf{1 0 0} \text { mil } \\
\text { habitantes (A) }\end{array}$ & $\begin{array}{c}\text { Razão médico/ } \\
\text { Necessidade da } \\
\text { microrregião } \\
\text { (B) }\end{array}$ & $\begin{array}{c}\text { Existente SUS } \\
\text { (C) }\end{array}$ & $\begin{array}{c}\text { Existente privado } \\
\text { (D) }\end{array}$ & $\begin{array}{c}\text { Resultado } \\
\text { (C+D-B) }\end{array}$ \\
\hline Angiologia & 1,5 & 1,83 & 0 & 1 & $-0,83$ \\
Cirurgia Vascular & 1,5 & 1,83 & 0 & 0 & $-1,83$ \\
Cardiologia & 6,5 & 7,91 & 9 & 1 & 2,09 \\
Dermatologia & 2,3 & 2,8 & 0 & 2 & $-0,8$ \\
Endocrinologia & 1,5 & 1,83 & 0 & 0 & $-1,83$ \\
Gastroenterologia & 2,5 & 3,04 & 0 & 0 & $-3,04$ \\
Proctologia & 2 & 2,43 & 0 & 0 & $-2,43$ \\
Neurologia & 3,5 & 4,26 & 3 & 0 & $-1,26$ \\
Neurocirurgia & 3,5 & 4,26 & 1 & 0 & $-3,26$ \\
Neurofisiologia & 3,5 & 4,26 & 0 & 0 & $-4,26$ \\
Oftalmologia & 4 & 4,87 & 1 & 1 & $-2,87$ \\
Ortopedia & 10 & 12,17 & 5 & 0 & $-1,17$ \\
Otorrino & 3 & 3,65 & 2 & 0 & $-2,65$ \\
Pneumologia & 3 & 3,65 & 1 & 0 & $-3,65$ \\
Urologia & 3 & 3,65 & 0 & 0 & $-0,22$ \\
Reumatologia & 1 & 1,22 & 0 & 1 & \\
\hline
\end{tabular}

Fonte: Gonçalves E, et al., 2021; dados extraídos do Datasus (BRASIL, 2020). 


\section{DISCUSSÃO}

O presente estudo identificou um desequilíbrio na distribuição de profissionais médicos em geral tanto nas microrregiões compreendidas na área de abrangência dos consórcios intermunicipais de saúde, como em uma mesma macrorregião. Esses dados corroboram com os achados do estudo Demografia Médica no Brasil, realizado pelo Conselho Federal de Medicina, que compreendeu etapas diferentes, utilizando-se dados extraídos de bases oficiais distintas, estudos sobre a formação médica e a oferta de graduação em medicina e residência médica e por meio de inquérito nacional sobre trabalho médico com amostra probabilística de médicos com registros nos 27 Conselhos Regionais de Medicina (SCHEFFER M, et al., 2020). Este estudo apresentou também 4 especialidades que juntas concentram cerca de $40 \%$ dos especialistas que são: Clínica Médica ( $11,3 \%$ do total de especialistas), Pediatria (10,1\%), Cirurgia Geral $(8,9 \%)$ e Ginecologia e Obstetrícia $(7,7 \%)$.

A pesquisa não encontrou resultados nas bases de dados algumas especialidades, como a cirurgia vascular e a proctologia com profissionais disponíveis no Cadastro Nacional de Estabelecimentos de Saúde (CNES), tanto para o Sistema Único de Saúde (SUS), quanto para o setor privado. Já a ortopedia apresentou déficit considerável em todas as microrregiões e, a cardiologia, índices acima do parâmetro expectado pela portaria ministerial (BRASIL, 2015). Os dados são provenientes do CNES, cujo registros possuem vinculação direta quanto as transferências de recursos financeiros que podem impactar na relação união, estado e municípios, havendo em qualquer tempo a obrigatoriedade de atualização permanente pelos gestores correspondentes em cada esfera.

Estudos avaliativos que se propõem a analisar a distribuição de profissionais no âmbito das regiões de saúde podem contribuir significativamente para a redução das iniquidades e na busca por respostas assistenciais em tempo oportuno na média e alta complexidade historicamente enfrentada. Em que pese esta análise associada aos Consórcios Intermunicipais de Saúde tem especial relevância, uma vez que estes dispositivos carregam em si a premissa de superação de vazios assistenciais e resolutividade assistencial, em que a análise pormenorizada destas conexões interessa demasiadamente a todos os entes consorciados.

São evidentes os desafios apresentados pelos municípios, como a dificuldade técnica, financeira e administrativa, que convergem consequentemente às dificuldades de atendimento pleno as demandas de saúde de que lhes são requisitadas diariamente, principalmente para os serviços de média e alta complexidade (LOBO-NETO FJS, 2014; GRIN EJ, et al., 2018).

Um estudo demonstrou que há dificuldades para o acesso de pacientes em especialidades como a cirurgia vascular, proctologia, geriatria, endocrinologia, neurologia e apontou a insuficiência também para ortopedia, neuropediatria, urologia, reumatologia, oftalmologia e otorrinolaringologia (SILVA CR, et al., 2017). E, no presente estudo, estas especialidades apresentaram distribuição iníqua entre as microrregiões de abrangência dos consórcios intermunicipais de saúde, fato que pode ser um dos motivos da dificuldade de acesso apontada no estudo supracitado. Impactos negativos quanto a inobservância destes cenários, como a indisponibilidade de profissionais especialistas, tem influenciado a assunção de responsabilidades pelo ente municipal, essas que extrapolam a aplicabilidade do mínimo constitucional preconizado pela Lei 141/2012 para os gastos com saúde, cuja ascensão principal se apresenta pelo provimento da média e alta complexidade dos municípios (BRASIL, 2012; SILVA CR, et al., 2017).

A responsabilização financeira refletida pelos municípios é resultado da descentralização sob a ótica deste ente, principalmente para as ações ambulatoriais, com maior representatividade dos gastos nos últimos anos (ROCHA CV, 2016).

Os dados apresentados nas tabelas de cada microrregião remontam a grande desigualdade ainda presente em cada território, quanto a concentração da maioria dos profissionais especialistas presentes nos municípios polos da microrregião, sendo uma das maiores dificuldades dos municípios consorciados, em especial, os de pequeno porte, reverberando na incapacidade da oferta integral à saúde aos munícipes (PINAFO E, et al., 2020). É corroborado pela literatura também a perenização dos investimentos e alocações de equipamentos de média e alta complexidade em um pequeno número de municípios, considerando a totalidade dos 5570 municípios do país, e, ainda, os desafios associados aos deslocamentos para o alcance destes equipamentos e pela busca de profissionais especialistas (VIACAVA F, et al., 2014). 
Neste contexto as políticas regionais na área da saúde implementadas ao longo do tempo trouxeram avanços expressivos, como a negociação entre os gestores, a expansão da rede, os investimentos e o planejamento, tudo isso visando a redução das desigualdades regionais (ALBUQUERQUE MV, et al., 2017). Contudo, é possível ainda identificar por meio de estudos que decisões políticas ainda se sobrepõe a critérios técnicos e evidências, como a fragilidade no monitoramento, dificultando ainda mais os avanços quando das negociações com os prestadores disponíveis (MEDEIROS CRG et al., 2017).

Estudos demonstraram que a taxa de médicos per capita aumenta com a renda local, requerendo uma busca pela equalização da renda regional per capita, de modo que, essa desigualdade distributiva dos médicos tenda a ser amenizada geograficamente, mas também indicam ainda que médicos mais jovens estão mais propensos a mudanças (VAN STRALEN ACS, et al., 2017; PIERANTONI CR, et al., 2019). Para mais, que a estrutura de ensino médico de um estado atrai médicos em busca de aperfeiçoamento, assim como a atração pelo fator econômico regional, associados ao papel também do conjugue, na decisão locacional do profissional médico (VAN STRALEN ACS, et al., 2017; PIERANTONI CR, et al., 2019).

Sendo assim, o diálogo permanente nas instâncias de gestão do SUS, sobre quais os modelos de formação profissional se quer construir, o fortalecimento dos atuais arranjos públicos e universais capazes de reduzir as iniquidades, a atenção as relações constituídas junto aos entes consorciados em cada localidade, os potenciais cenários alcançáveis e possivelmente compartilhados por todos os consórcios da área de abrangência de uma macrorregião possa, em um curto espaço de tempo, apresentar resultados significativos para a equalização econômica destas relações (CARVALHO MS e SOUSA MF, 2013; ALMEIDA PF, et al., 2019).

Espera-se assim, o cuidado integral e resolutivo para os usuários do SUS, cuja negociação, construção consensual e superação de obstáculos são fundamentais para a consolidação da regionalização, em que os consórcios intermunicipais de saúde estão presentes e devem compartilhar do protagonismo assistencial (MOREIRA MR, et al., 2017). Desta forma, faz-se necessária a compreensão que a distribuição geográfica dos médicos apresenta influência direta para o bem-estar social da população, em que o entendimento dos determinantes desta distribuição deve ser objeto de análise e discussão constante nas instâncias de gestão do SUS, em especial, dos consórcios intermunicipais de saúde.

\section{CONCLUSÃO}

Conclui-se que, há grande variabilidade entre as microrregiões na área de abrangência dos consórcios intermunicipais de saúde existentes, quanto a distribuição de profissionais especialistas dentro de uma mesma microrregião, considerando a inexistência de alguns profissionais com vínculo para o SUS e para o setor privado. Todavia, como limitação deste estudo, aponta-se a possibilidade da falta de atualização oportuna pelos gestores municipais dos dados inseridos no CNES. Fora isso, acredita-se que o presente estudo possa contribuir para a indução de discussões que balizem o alinhamento e a adoção de políticas em contraponto as dificuldades de provimento de profissionais em variadas categorias, nas áreas de abrangência dos consórcios intermunicipais de saúde, estabelecendo condições reais de fixação, para corresponder ao financiamento justo e adequado, fortalecendo assim, as regiões de saúde, as redes de cuidado, a otimização dos recursos e a gestão do SUS.

\section{REFERÊNCIAS}

1. ALBUQUERQUE MV, et al. Desigualdades regionais na saúde: mudanças observadas no Brasil de 2000 a 2016. Ciência \& Saúde Coletiva, 2017; 22(4): 1055-1064.

2. ALMEIDA PF, et al. Redes regionalizadas e garantia de atenção especializada em saúde: a experiência do Ceará, Brasil. Ciência \& Saúde Coletiva, 2019; 24(12): 4527-4540.

3. ARAÚJO CEL, et al. Os municípios brasileiros e os gastos próprios com saúde: algumas associações. Ciência \& Saúde Coletiva, 2017; 22(3): 953-963.

4. ARRETCHE MTS, MARQUES EC. Condicionantes locais da descentralização das políticas de saúde. In: Políticas públicas no Brasil. Rio de Janeiro: Fiocruz, 2003.

5. BRASIL. Constituição (1988). Emenda Constitucional n. 98, de 06 de dezembro de 2017. Altera o Ato das Disposições Constitucionais Transitórias, para instituir o Novo Regime Fiscal, e dá outras providências. Brasília, DF, 2017. 
6. BRASIL. Lei № 141, de 06 de abril de 2012. Regulamenta o § 30 do art. 198 da Constituição Federal para dispor sobre os valores mínimos a serem aplicados anualmente pela União, Estados, Distrito Federal e Municípios em ações e serviços públicos de saúde; estabelece os critérios de rateio dos recursos de transferências para a saúde e as normas de fiscalização, avaliação e controle das despesas com saúde nas 3 (três) esferas de governo; revoga dispositivos das Leis nos 8.080, de 19 de setembro de 1990, e 8.689, de 27 de julho de 1993; e dá outras providências, 2012.

7. BRASIL. Ministério da Saúde. Departamento de Informática do Sistema Único de Saúde (Datasus), 2020.

8. BRASIL. Ministério da Saúde. Portaria n. 1.631, de 1ำ de outubro de 2015. Aprova critérios e parâmetros para o planejamento e programação de ações e serviços de saúde no âmbito do SUS. Diário Oficial da União. Brasília-DF, 01 de out. p. 38, 2015.

9. CARVALHO ALB, et al. Regionalização no SUS: processo de implementação, desafios e perspectivas na visão crítica de gestores do sistema. Ciência \& Saúde Coletiva, 2017; 22(4): 1155-1164.

10. CARVALHO MS, SOUSA MF. Como o Brasil tem enfrentado o tema provimento de médicos? Interface - Comunicação, Saúde, Educação, 2013; 17(47): 913-926.

11. CONFEDERAÇÃO NACIONAL DE MUNICÍPIOS. Consórcios Públicos Intermunicipais: Uma Alternativa à Gestão $\begin{array}{lcc}\text { Pública. } & \text { Brasília, } & \text { Disponível } \\ \text { https://www.cnm.org.br/cms/biblioteca antiga/Consórcios\%20públicos\%20intermunicipais\%20- }\end{array}$ \%20Uma\%20alternativa\%20à\%20gestão\%20pública.pdf. Acessado em 14 de dezembro de 2020.

12. CONTARATO PC, et al. Crise e federalismo: tendências e padrões regionais das receitas e despesas em saúde dos estados brasileiros. Ciência \& Saúde Coletiva, 2019; 24(12): 4415-4426.

13. FLEXA RGC, BARBASTEFANO RG. Consórcios públicos de saúde: uma revisão da literatura. Ciência \& Saúde Coletiva, 2020; 25(1): 325-338.

14. FUNCIA FR. Subfinanciamento e orçamento federal do SUS: referências preliminares para a alocação adicional de recursos. Ciência \& Saúde Coletiva, 2019; 24(12): 4405-4415.

15. GIRARDI SN, et al. Índice de escassez de médicos no Brasil: estudo exploratório no âmbito da Atenção Primária. In: PIERANTONI CR, et al. O trabalho em Saúde: abordagens quantitativas e qualitativas. Rio de Janeiro: Cepesc/IMS/UERJ, ObservaRH, 2011; 171-186.

16. GRIN EJ, et al. Sobre Desconexões e Hiatos: Uma Análise de Capacidades Estatais e Finanças Públicas em Municípios Brasileiros. Cadernos Gestão Pública e Cidadania, 2018; 23(76): 312-336.

17. GRZELZAK M, et al. A IMPORTÂNCIA DOS CONSÓRCIOS INTERMUNICIPAIS DE SAÚDE. South American Development Society Journal, 2017; 3(07): 196-205.

18. JULIÃO KS, OLIVEIRA C. Cooperação intergovernamental na política de saúde: a experiência dos consórcios públicos verticais no Ceará, Brasil. Cadernos de Saúde Pública, 2020; 36(3).

19. LEAL EMM, et al. Razões para a expansão de consórcios intermunicipais de saúde em Pernambuco: percepção dos gestores estaduais. Saúde e Sociedade, 2019; 28(3): 128-142.

20. LIEBEL G. Fatores Associados aos Gastos com a Produção Ambulatorial em média complexidade (Dissertação de mestrado). Universidade Federal de Juiz de Fora, Juiz de Fora, MG, 2016; 78 p.

21. LOBO-NETO FJS. Democracia, federalismo e centralização no Brasil. Trabalho, Educação e Saúde, 2014; 12(2): 459462.

22. MEDEIROS CRG, et al. Planejamento regional integrado: a governança em região de pequenos municípios. Saúde e Sociedade, 2017; 26(1): 129-140.

23. MENDES W, et al. Consórcios intermunicipais de saúde: uma reflexão sobre dicotomia entre administração pública e privada. Nucleus, 2019; 16(1): 195-204.

24. MOREIRA MR, et al. Obstáculos políticos à regionalização do SUS: percepções dos secretários municipais de Saúde com assento nas Comissões Intergestores Bipartites. Ciência \& Saúde Coletiva, 2017; 22(4): 1097-1108.

25. ORGANIZAÇÃO MUNDIAL DA SAÚDE (OMS). Estatísticas Sanitária Mundiais Genebra: OMS, $2012 ; 178$ p.

26. PIERANTONI CR, et al. Graduação em saúde: oferta e estratégias para o fortalecimento da regionalização do Sistema Único de Saúde. Cad Saude Publica, 2019; 13(35Suppl 2): e00066018.

27. PINAFO E, et al. Problemas e estratégias de gestão do SUS: a vulnerabilidade dos municípios de pequeno porte. Ciência \& Saúde Coletiva, 2020; 25(5): 1619-1628.

28. ROCHA CV. A cooperação federativa e a política de saúde: o caso dos Consórcios Intermunicipais de Saúde no estado do Paraná. Cadernos Metrópole, 2016; 18(36): 377-399.

29. SANTOS L, CAMPOS GWS. SUS Brasil: a região de saúde como caminho. Saúde e Sociedade, 2015; 24(2): 438446.

30. SANTOS-NETO JA, et al. Avaliação da eficiência técnica em saúde dos municípios da região de saúde Rota dos Bandeirantes do estado de São Paulo, Brasil. Ciência \& Saúde Coletiva, 2019; 24(10): 3793-3803.

31. SCHEFFER M, et al. Demografia Médica no Brasil 2020. São Paulo: Faculdade de Medicina da Universidade de São Paulo, Conselho Federal de Medicina, 2020.

32. SILVA CR, et al. Dificuldade de acesso a serviços de média complexidade em municípios de pequeno porte: um estudo de caso. Ciência \& Saúde Coletiva, 2017; 22(4): 1109-1120.

33. UNIVERSIDADE FEDERAL DE MINAS GERAIS. Dimensionamento da estrutura e dinâmica do mercado de trabalho em Atenção Básica em Saúde. Belo Horizonte: EPSM/NESCON/FM/UFMG, 2014. Disponível em: http://epsm.nescon.medicina.ufmg.br/epsm/Relate Pesquisa/Dimensionamento\%20da\%20estrutura\%20e\%20dinâmi ca\%20do\%20mercado\%20de\%20trabalho\%20em\%20AB\%20em\%20Saúde\%20(Rel\%20Final).pdf. Acessado em 8 de dezembro de 2020.

34. VAN STRALEN ACS, et al. Percepção de médicos sobre fatores de atração e fixação em áreas remotas e desassistidas: rotas da escassez. Physis, 2017; 27: 147-72.

35. VIACAVA F, et al. Relatório de pesquisa sobre internações na esfera municipal. Ministério da Saúde, Fundação Oswaldo Cruz, 2014. 